\title{
TB OR XDR TB, SHOULD THAT BE THE QUESTION?
}

\author{
Andrew Black, BSc, MB BCh, FCP (SA), Cert Pulm (SA), FCCP (USA) \\ Department of Medicine, Chris Hani Baragwanath Hospital, fohannesburg
}

The emergence and recognition of extensively drug-resistant tuberculosis (XDR TB) in South Africa led to a wave of panic fuelled by media hype. The response was predictable: meetings, circulars and finally a seven-point emergency action plan. ${ }^{1}$ Fortunately Mycobacterium tuberculosis (TB) replicates slowly, as very little seems to have happened in the 5 months since the proposal of the seven-point plan.

The seven-point emergency action plan for XDR TB is based on the seven-point plan devised for MDR TB in the 1990s. ${ }^{2}$ The emergence of XDR TB is a consequence of the earlier plan's failure. Are we doing any better this time?

\section{THE SEVEN-POINT PLAN}

\section{RAPID SURVEYS TO ASSESS THE CURRENT PREVALENCE OF XDR TB}

The only comprehensive survey for South Africa conducted during the current HIV pandemic produced figures of 39\% for MDR TB and 6\% for XDR TB. ${ }^{3}$ These figures were for a single small region in KwaZulu-Natal, and until comprehensive surveys are conducted nationally one can only speculate on the true extent of the problem in South Africa.

Hospital budgets are stretched and the further financial burden of routine culture and drug sensitivity testing on all cases of suspected TB cannot be borne by individual institutions. Limiting TB culture and susceptiblity testing of patients with persistent acid-fast bacilli (AFB) and retreatment cases in accordance with the National Guidelines will not provide the information required. In the KwaZulu-Natal series the median time from sputum collection until death for XDR TB cases was 16 days, leaving no time to fail treatment. Fiftyfive per cent of the XDR TB cases had no history of prior TB. Central funding is required for the culture and drug sensitivity testing of all suspected TB cases nationwide to define the true extent and location of the problem.

Any intervention aimed at addressing a problem the extent and distribution of which is unknown will not be able to be assessed and may squander time and money. The cost of

XDR TB is defined as an isolate of TB resistant to isoniazid (INH) and rifampicin (multidrug-resistant tuberculosis) plus any fluoroquinolone and at least one of three injectable second-line agents (i.e. amikacin, kanamycin or capreomycin). conducting a comprehensive national surveillance programme may be better spent on improving the current TB treatment programmes. This strategy will decrease drug resistance even if the extent of the problem is not known.

\section{ENHANCE LOCAL LABORATORY CAPACITY TO CARRY OUT CULTURE AND DRUG RESISTANCE TESTING}

Laboratories are already strained by the current load of specimens and need to honestly assess their capacity to deal with a much larger volume if routine culture and susceptibility testing is to be advocated. Quality assurance and reproducibility is imperative so that clinicians may have confidence in the laboratory results. Laboratories should have the expertise and equipment available to perform the services they offer.

There is currently no system whereby clinicians are provided with a monthly list of culture-positive results for their hospital. Lack of this seemingly basic information means that hospitals are unable to identify and trace culture-positive patients who have not returned for follow-up.

Laboratory services need to take more responsibility with regard to the notification of clinicians or hospitals of positive TB culture results. A step in the right direction is the fact that the laboratory now contacts the clinician if a culture is found to be MDR TB. This needs to be extended to all positive TB cultures.

\section{INCREASED TRAINING OF PUBLIC HEALTH STAFF TO IDENTIFY, INVESTIGATE AND TREAT XDR OUTBREAKS}

Training and education needs to involve staff who manage TB patients and needs to focus on the control of all TB and not just XDR TB, as our TB incidence of 718/100 $000^{4}$ suggests that 
South Africa's national TB control programme is not functioning. Without routine surveillance and a central point for all culture results, outbreaks of XDR TB will be missed because no specific clinical features have yet been identified that may alert clinicians to suspect XDR TB.

\section{IMPLEMENTATION OF INFECTION CONTROL PRECAUTIONS}

Infection control should be more than just a sign on the door. Public sector hospitals often face a large TB burden, and are required to manage an excessive number of patients with limited resources. As an illustration, a large tertiary hospital in Gauteng Province registered 6992 cases of TB for 2006 - just under 20 new TB cases daily (personal communication). At this hospital over 100 medical patients are admitted daily via a 32bed admission ward. Only 6 single-bed cubicles are available for the isolation of general medical patients. Medical staff at this hospital have no access to P95 masks. There are no ultraviolet lights or ventilatory control systems in any of the medical wards. Given the patient numbers and available facilities, no meaningful infection control is possible.

\section{INCREASED RESEARCH SUPPORT FOR DRUGS TO TREAT XDR TB}

We currently have highly effective short-course therapy available to treat TB. In South Africa nationally we achieve only a 54\% cure rate for new smear-positive TB cases. ${ }^{4}$ Some provinces are unable to cure a third of their TB cases.

There is an urgent need to develop drugs to treat the few patients with confirmed XDR TB. However, more drugs and more complex regimens will be of little use overall until we start to treat new smear-positive cases adequately. The introduction of new drugs into an already failing programme is likely to result in more resistant cases than they would treat.

\section{DEVELOPMENT OF RAPID DIAGNOSTIC TESTS FOR TB}

Cost will be the deciding factor when these tests are developed. Improved diagnosis is, however, of little benefit if the TB diagnosed is not treated adequately and cured.

\section{ACCESS TO ANTIRETROVIRAL THERAPY}

Antiretroviral therapy (ART) decreases the incidence of TB dramatically. This decrease is dependent on the absolute CD4 count. ${ }^{5}$ To decrease the incidence of TB in South Africa we need to increase voluntary counselling and testing (VCT), clear backlogs at ART clinics, shorten the time from HIV diagnosis to commencement of ART, and campaign for a CD4 count higher than the current 200 to qualify for ART. ART in prisons, which are notorious for high TB incidence, is essential.

Other considerations include assessing whether we are not inadvertently encouraging resistance through well-meaning practices. The use of fluoroquinolones not only delays the diagnosis of TB but may promote the resistance to this class of second-line TB agents. ${ }^{6}$ These findings are worrying given the liberal use of ciprofloxacin for diarrhoea in HIV-positive patients.
Isoniazid (INH) prophylaxis is highly effective, but the risk of inducing resistance needs to be seriously considered, particularly in a population group where proving a patient does not have active TB is as difficult as proving they do.

There are still questions that need to be answered with regard to the current treatment regimens in our patient population. Rifampicin may not be as well absorbed when part of fixeddrug formulations as when administered as a single agent. ${ }^{7}$ Rifampicin's absorption is significantly decreased in HIV seropositive patients, particularly those with chronic diarrhoea. ${ }^{8}$ The possible induction of widespread rifampicin mono-resistance would make short-course therapy ineffective. Large pharmacokinetic and pharmacodynamic studies are needed in our patient population to determine whether we are achieving the desired drug concentrations.

$X D R T B$ is the end of a spectrum of a man-made problem and a result of poor TB treatment. The MDR/XDR TB problem can be overcome (even without quantifying it) by treating TB and HIV adequately, ensuring patient compliance with directly observed therapy, and practising adequate infection control measures in our clinics, hospitals, prisons and communities. These measures have been shown to not only decrease overall TB incidence but also decrease the prevalence of MDR TB in both HIV-seropositive and seronegative patients. ${ }^{9}$

\section{REFERENCES}

SA Health Info, report from the expert consultation on drug-resistant tuberculosis (Sept 7-8, 2006). http://www.sahealthinfo.org/tb/expert.htm (accessed 8 January 2006).

2. Van Rie A, Enarson D. XDR tuberculosis: an indicator of public health negligence. Lancet 2006; 368: 1554-1556.

3. Gandhi NR, Moll A, Sturm AW, et al. Extensive drug-resistant tuberculosis as a cause of death in patients co-infected with tuberculosis and HIV in a rural area in South Africa. Lancet 2006; 368: 1575-1580.

4. Global tuberculosis control: surveillance, planning, financing. WHO report 2006. www.who.int/tb (accessed 10 January 2007).

Lawn SD, Myer L, Bekker LG, Wood R. Burden of tuberculosis in an antiretroviral treatment programme in Sub-Saharan Africa: Impact on treatment outcomes and implication for tuberculosis control. AIDS 2006; 20 (12): 1605-1612.

6. Wang JY, Hsueh PR, Jan IS, et al. Empirical treatment with a fluoroquinolone delays the treatment for tuberculosis and is associated with a poor prognosis in endemic areas. Thorax 2006; 61(10): 903-908.

Iseman MD. Tuberculosis chemotherapy, including directly observed therapy. In: Iseman MD, ed. A Clinician's Guide to Tuberculosis. Philadelphia: Lippincott Williams \&t Wilkins, 2000: 271-322.

8. Gurumurthy P. Decreased bioavailability of rifampin and other antituberculosis drugs in patients with advanced human immunodeficiency virus disease. Antimicrob Agents Chemother 2004; 48 (11): 4473-4475.

9. Frieden TR, Fujiwara PI, Washko RM, Hamburg MA. Tuberculosis in New York City - turning the tide. N Engl J Med 1995; 333: 229-233.

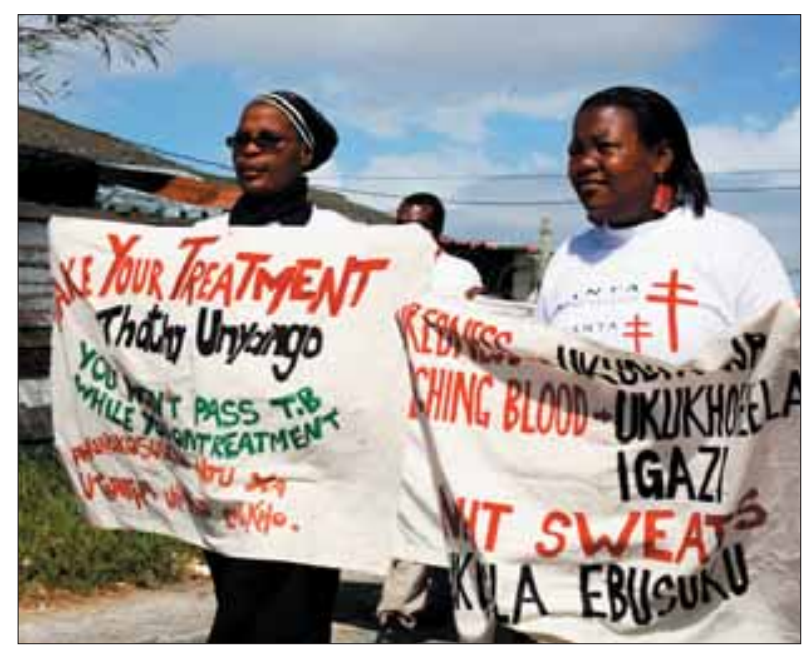

